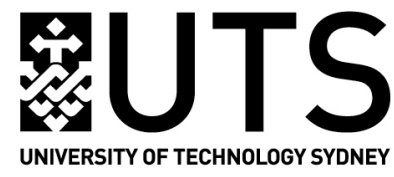 \\ PAM Review
}

Subject 68412 www.uts.edu.au

\section{Using photonic cooling systems to improve the efficiency of photovoltaic cells and their means of electricity production Alice Gordon ${ }^{1}$, Ben Cross ${ }^{2}$ and Oliver Evans ${ }^{3}$}

\author{
University of Technology Sydney, Faculty of Science, PO Box 123, Ultimo NSW 2017, Australia \\ 1 Alice.r.gordon@student.uts.edu.au \\ 2 Benjamin.j.cross@student.uts.edu.au \\ 33liver.a.evans@student.uts.edu.au \\ *Author to whom correspondence should be addressed.
}

DOI: http://dx.doi.org/10.5130/pamr.v5i0.1498

\begin{abstract}
Photovoltaic (PV) systems generate electricity from the abundance of solar energy provided by the sun, making them a significant technology in utilising clean, renewable energy. It is in our best interest to achieve the maximum possible electrical output from these systems.

$\mathrm{O}$ : Solar photovoltaic (PV) systems are a effective technology to resource clean energy, it is in our best interest to achieve the maximum possible electrical output from these cells.

Through this meta-study we discuss how the efficiency of photovoltaic systems can be optimised using photonic cooling systems (PCS). The use of laser and doppler cooling upon photovoltaic (solar) cells combat the thermodynamic property of semiconductors where increases in temperature result in a decrease in electroconductive efficiency. PCS' ability to bring materials to the $\mathrm{mK}$ range theoretically improves PV systems efficiency by more than double the current practical results. However, we show the inability for semiconductors to function at such low temperatures resulting in such cells acting as insulators in the $\mathrm{mK}$ range. Through this meta-study we have researched efficiency of PV cells and PCS through multiple paper analysis. Using databases such as Scopus and Nature where we recognise highly cited journals/papers while focusing upon PV and PCS as search parameters. Linking between their temperature, efficiency and success during practical testing.
\end{abstract}

Keywords: Photonic thermodynamics, Photovoltaic systems; Photonic cooling systems; Efficiency; Semiconductor; Doppler cooling 


\section{Introduction}

Modern photovoltaic (PV) cells have huge potential within their capabilities as far as theoretical efficiency and energy output is concerned, these systems have proven to be an invaluable method of renewable power generation [1]. The maximum single cell p-n junction PV cell efficiency of 33.7\% [2] is significantly higher than the standard $15 \%$ of current commercial cells efficiency displaying room for expansion within this field. However, multi-junction (MJ) photovoltaic cells are more useful for our study, the optimum efficiency of these reaching up to a theoretical value of $86.8 \%$ whereas currently values upwards of only $46 \%$ efficiency have been established under lab conditions under concentrated sunlight [3]. The limitations of modern PV technologies show how the efficiency of PV cells and overall power output decreases as the temperature of cells rises (recorded loss of $0.258 \%$ for every degree above $300 \mathrm{~K}$ in Panasonic VBHN330SA16 solar panels or on average 0.4\%) [4]. This efficiency loss is due to the decrease in potential differences between two energy states/temperatures. The power generation occurs as a photon from sunlight excites an electron from one state to another. This ejection of the electron from a valance band (VB) to the conduction band (CB) where it is free to form part of an electric current therefore generating electricity [5].

PV cells work in accordance to Carnot system mechanics [6]. Processes within the Carnot cycle make two key temperatures vital to the efficiency of the process:

$$
\left.\eta(\%)=1-\mathrm{T}_{\mathrm{C}} / \mathrm{T}_{\mathrm{H}} \times 100 \% \text { (eqn. } 1\right)
$$

Efficiency of a Carnot process $(\eta)$ increases when the difference between the temperature of the cell $\left(\mathrm{T}_{\mathrm{C}}\right)$ and temperature of the sun $\left(\mathrm{T}_{\mathrm{H}}\right)$ is greater. With the temperature of the sun being approximately $5778 \mathrm{~K}$ [7], if temperatures of the cell were reduced to less than $100 \mathrm{~K}$, theoretically, efficiencies should occur at greater than $98 \%$ (according to eqn.1). Should this theory prove correct by experimental results, laser cooling would be an excellent candidate to achieve such low temperatures. While this efficiency theory is sound at a basic level, the thermodynamic nature of the PV system and semiconductors in general does not match [8].

Effect of temperature upon Open Circuit Voltage of a photovoltaic cell

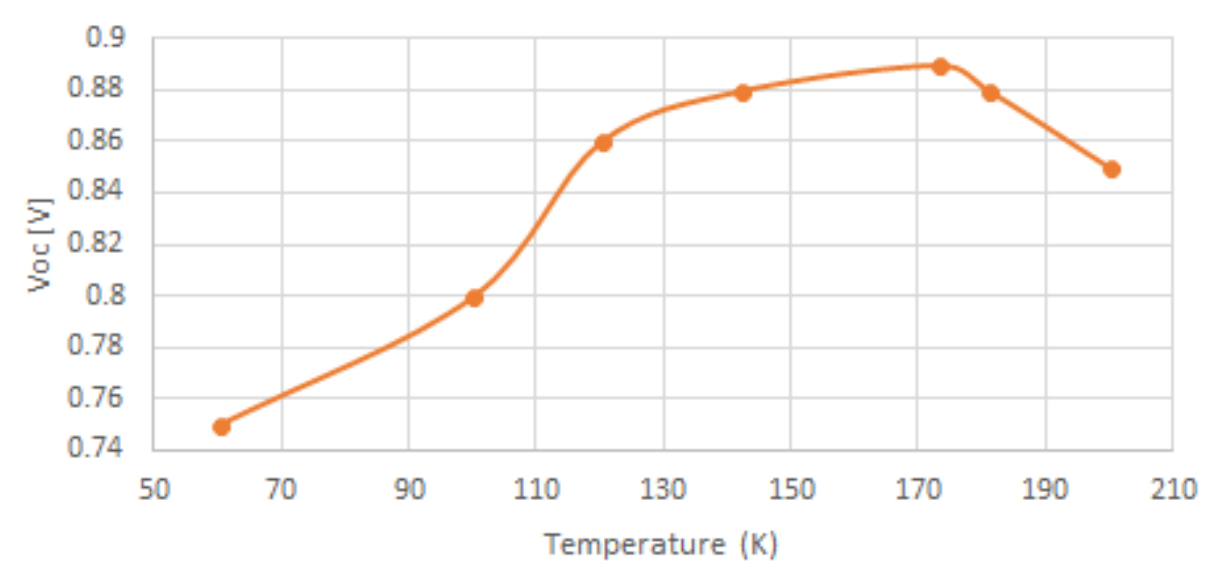

Figure 1. Displaying the difference that cryogenic temperatures has upon the $\mathrm{V}_{\mathrm{oc}}$ of solar cells. $\left(\mathrm{V}_{\mathrm{oc}}\right.$ relates to the voltage output before a load is applied to the cell [9]) 
PV systems function as a reversible Carnot engine in accordance to the second law of thermodynamics [10]. The entropy of the system exists where the system must function with as little absorption or generation as possible to achieve maximum output, as entropy can only be transferred as heat and cannot be absorbed [11].

Theoretically, if a PV system is absorbing heat from a source as powerful as the sun, a Carnot system's efficiency should increase as the temperature approaches $0 \mathrm{~K}$ [eqn.1]. Because of this correlation, methods of supercooling have gathered interest so that a maximum efficiency can be achieved.

Laser cooling devices can cool systems to temperatures of the $\mathrm{mK}$ range[12], making them a noteworthy method to apply to PV cells if experiment matches the theoretical results. Methods of photonic cooling such as Doppler cooling slows down the movement of particles by using light oscillating at similar frequencies to atoms to absorb the photons, gradually losing momentum as particles absorb the momentum from oncoming photons in a destructive interference [12]. The gradual loss of kinetic energy at an atomic level causes the atoms to drop in temperature.

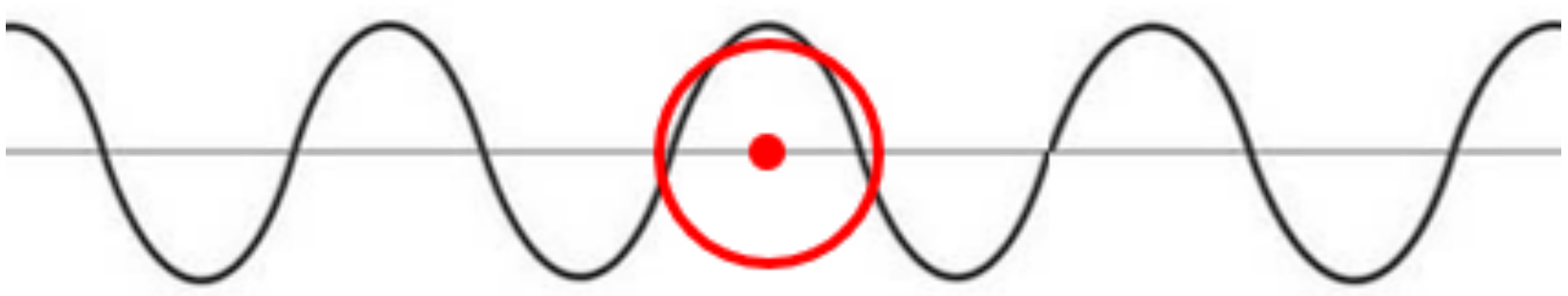

Figure 2. Doppler effect on a stationary atom. Incident light is not effected by doppler effect and the frequency does not match that of the atom and is therefore unlikely to be absorbed [13].
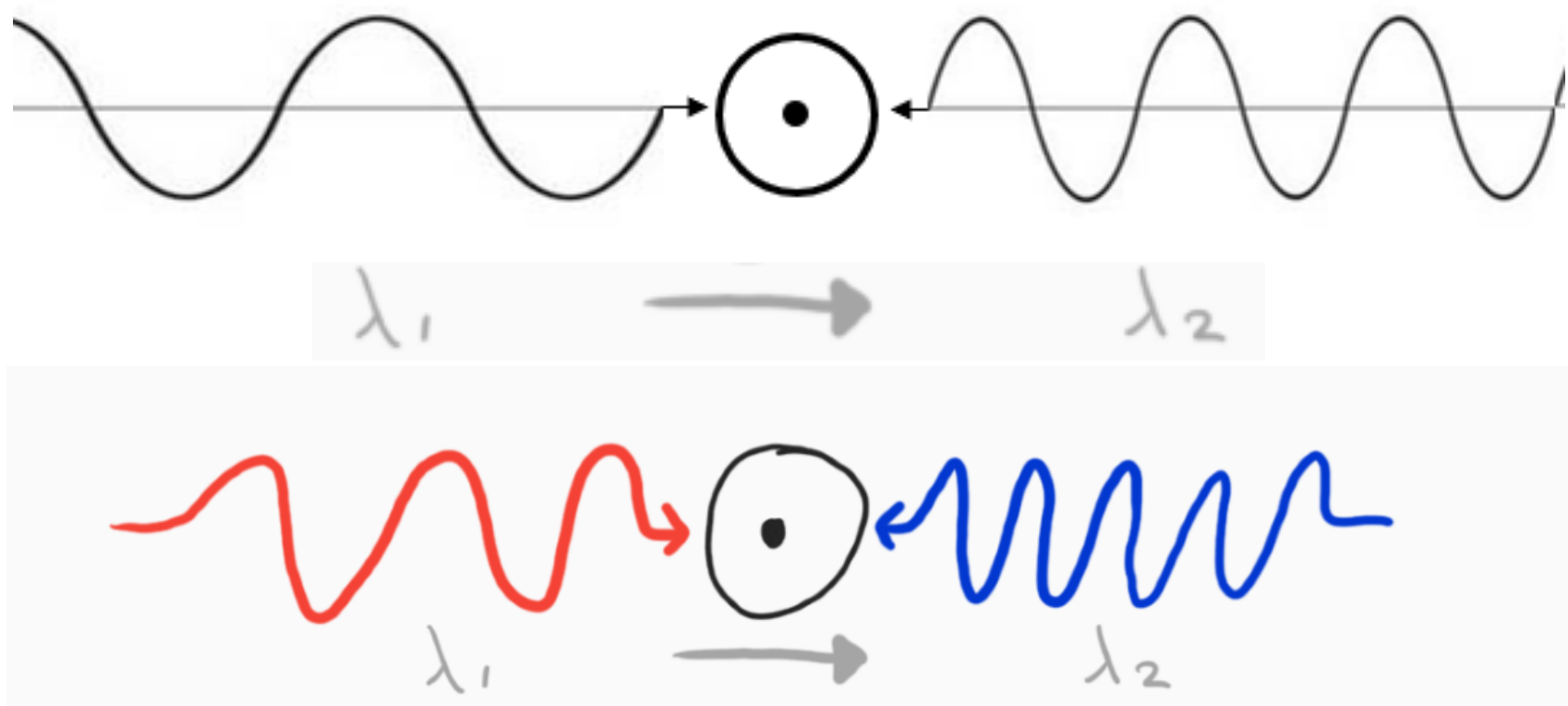

Figure 3. Visual representation of how incident lasers appear shifted due to the doppler effect on a moving electron. Incident rays from in front $\left(\lambda_{1}\right)$ appear to oscillate at a higher frequency (red shifted) and rays from behind $\left(\lambda_{2}\right)$ appear as a lower frequency (blue shifted). 


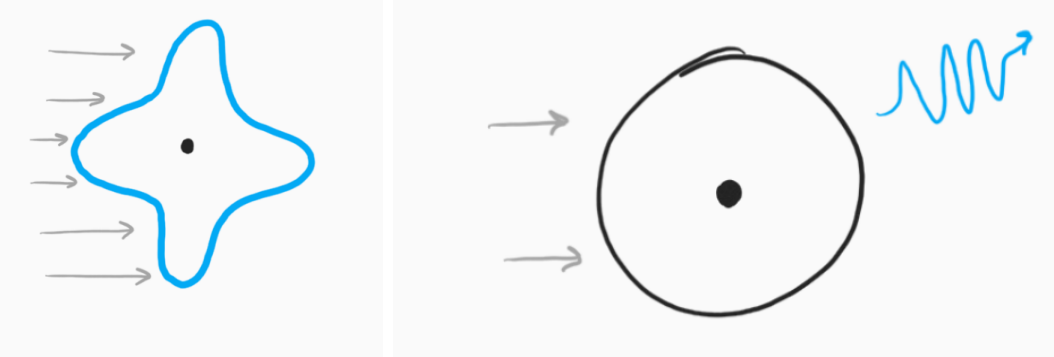

Figure 4,5. Excited atoms emit photons and lose momentum in the process.

Doppler cooling can bring the temperature of the atoms to their doppler temperature which is calculated through the equation.

$$
T_{\text {Doppler }}=\hbar \gamma /\left(2 k_{B}\right)[\text { eqn.2][13] }
$$

Where, $\mathrm{T}_{\text {doppler }}$ is the minimum possible temperature achievable for solids using this method of laser cooling. ' $\mathrm{k}_{\mathrm{B}}$ ' represents the Boltzmann constant $k=\frac{R}{N_{\mathrm{A}}}$. [14], 'h' is a reduced Plank's constant and 'y' corresponds to the natural linewidths. Dependent upon une varying dopant concentration, semiconductors have been shown to reach close to $60 \mathrm{~K}$ through the use of this cooling [15].

However, experimental results in applications towards semiconductors show a plateau in efficiency as temperatures arrive below 100K [16]. The semiconductors become inoperative as PV cells and lack the energy to excite electrons into the conducting band to generate an electrical circuit. Methods such as laser cooling are not a suitable method to increase the efficiency of PV cells because atoms with low energies are incapable of being excited to cross the band gap in a cell, making the need for super cooling of less than $100 \mathrm{~K}$ inefficient to run as it reaches beyond the maximum efficiency threshold.

We have focused on temperature as a variable for this report. As is known that the increase of temperature depletes PV cells efficiency. This increase in temperature is due to the vast amount of energy required to allow the conversion of sunlight into storable energy. What is often looked past is the increase in efficiency while the temperature of the PV cell decreases, this is true due to Silicon and Gallium's bandgaps increasing relative to their temperature[17]. This means the electrons which are attempting to climb bands do not have the sufficient energy to complete the phase increases decreasing the voltage out of the PV cell [18]. Due to this natural phenomenon we have decided to analyse what effect of temperatures reaching below the (regular range) but into the $\mathrm{mK}$ range. We have shown how to achieve this near absolute zero temperature range using Doppler laser cooling. However, as we discuss this supposably linear relationship between efficiency and temperature plateau's towards the low end of the temperature scale. 
Little research exists that evaluate the peak temperature efficiencies of PV cells and their potential to boost power generation. If cooler conditions were combined with other properties to boost efficiency and output such as doping, the expectations of future solar cells have potential to be significantly greater than the PV cells of the modern day.

\section{Methods}

The focus of this meta study was initially to investigate the applicability of photonic cooling devices to improve the efficiency of photovoltaic cells. Research databases such as Scopus, arXiv, nature, google scholar, and UTS library were consulted to search for a relationship between the temperature and the efficiency of the photovoltaic cell. These databases were filtered using a combination of keywords such as high photovoltaic systems, photonic cooling systems, and efficiency, to determine what relationship existed between the efficiency and temperature of the photovoltaic solar cell. Desirable articles originally focused on energy production of solar cells at near 0K temperatures. Experimental data for this was not found so searches become broader to find the relationship at below freezing.

We looked for articles that were detailed in our focus of study and had results which correlated with others to improve credibility. The data from the accepted studies included efficiency and temperature graphs as well as efficiency and band gap energy in relation to temperature.

In search for more information in applications to PV systems proved limited, so research was expanded to include supercooling applications towards semiconductors as they function similarly to PV systems with VB-CB energy activation.

\section{Results and Discussion}

\subsection{Efficiency of a photovoltaic cell vs temperature within a standard range}

From figure 5 it is apparent that there is a linear relationship between efficiency and temperature within normal operating conditions. It would be assumed that extrapolating the graphs, the linear trend would continue in both directions. Although we can interpret this trend as a decrease in temperature resulting in optimised efficiency, we are only interested in if this trend continues to cryogenic temperatures.

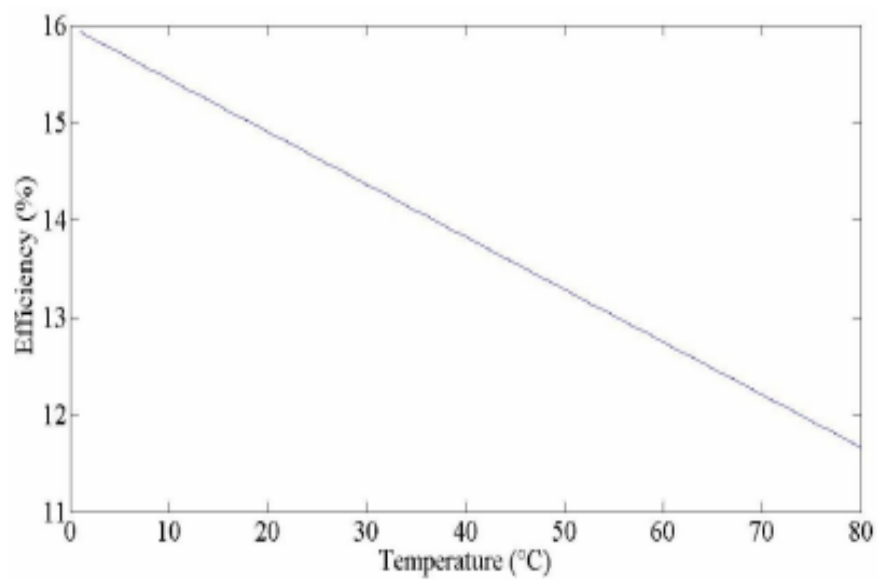

Figure 5. Efficiency of a photovoltaic cell vs temperature within a 'standard' range. Displaying the linear relationship between these two variables. [19] 


\subsection{Bandgap's Effect on Efficiency}

Figure 6 shows the effect of bandgap on the efficiency over a range of temperatures. The graph illustrates a bandgap of approximately $1.2 \mathrm{eV}$ and at a temperature of $50 \mathrm{~K}$ is where a the PV cells tested is at the highest efficiency. As temperature increases the efficiency is reduced, there is a negative effect to the changes in bandgap, particularly the smaller values.

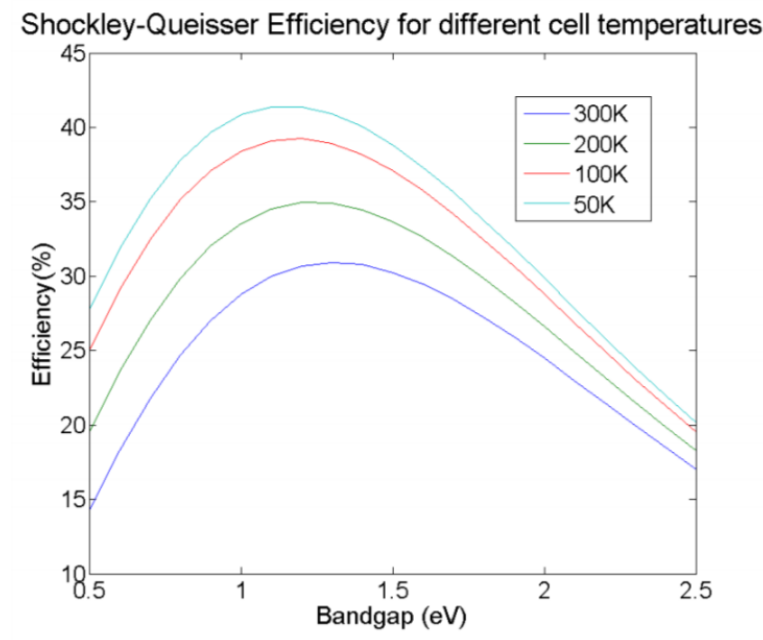

Figure 6. Showing the effect of bandgap upon efficiency and the effect of low temperatures upon the different bandgaps.[16]

\subsection{Efficiency vs Temperature from $50 \mathrm{~K}$ to $300 \mathrm{~K}$}

Figure 6 also states a peak efficiency at 50K however both figures lack experimental results as to how PV cells are affected by even lower cryogenic temperatures. Figure 7 has a more specific array of data making it easier to predict a trend for temperatures approaching absolute zero. As temperatures decrease, experimental data varies from theoretical as it encounters a limitation that is not accounted for in the theoretical hypothesis.

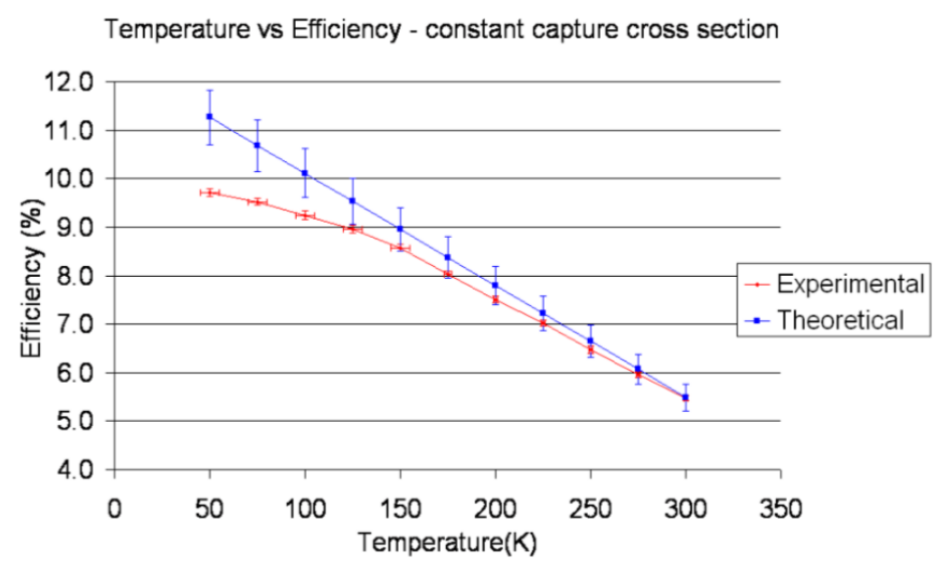

Figure 7. Experimental and theoretical efficiency vs Temperature at a cryogenic scale [16] 
Figure 8 is very similar to figure 9's voltage vs temperature curve although it is shifted significantly to a higher value of T. Figure 8 plateaus at $225 \mathrm{~K}$. This different turning point is due to the different type of PV cell used for the experiment and so happens to peak much lower than the given value of figure 9.

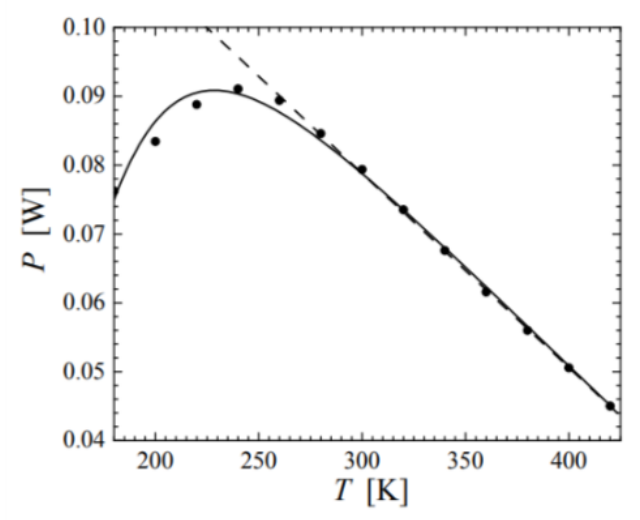

Figure 8. Experimental (circles) and theoretical (lines) temperature dependence of the photo-conversion power of HIT cells (heterojunction with intrinsic thin layer) [8]

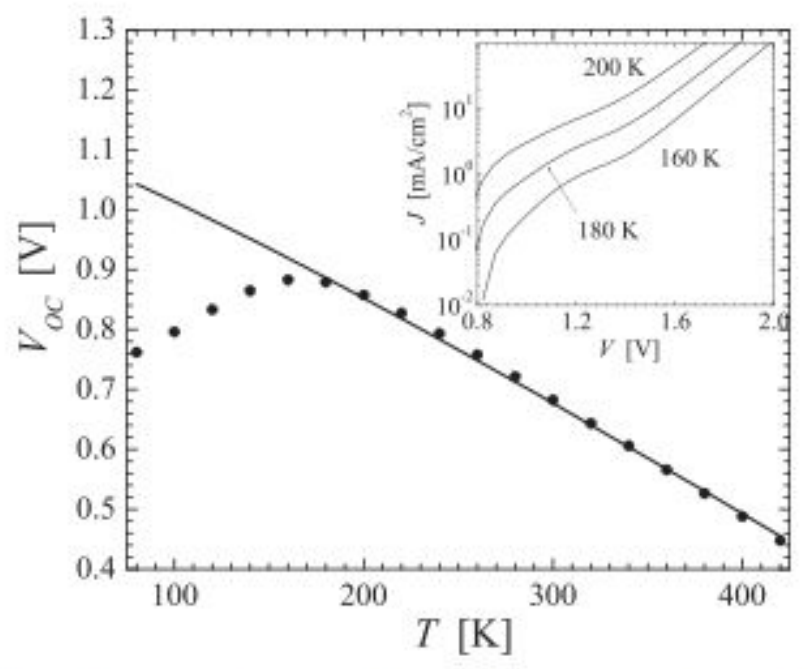

Figure 9. Experimental (circles) and theoretical (line) temperature dependence of the open-circuit voltage of PV cells. Inset displays the current-voltage curves at different the different low temperatures

Another reason why Doppler cooling has not been implemented through many photovoltaic devices is due to the huge amount of energy needed which is associated with; the manufacture of the PV cells combined with the laser cooling device and the necessity to keep to keep this technology operational[19]. In addition to the decrease of power output vs input from the solar cells when being doppler cooled to a fraction of a Kelvin, the overall energy consumption far outweighs the energy produced, making this method of optimising PV cells non-feasible when compared to the electrical production gain you would achieve[21]. The large expenses associated with this method of cooling could be combatted through the discovery/creation of a different photovoltaic cell (could be the base semiconductor or a different dopant material) where the valence to conduction band is easier to cross without negating the positive attributes. Similarly, if there was an easier way to house the laser cooling 
system without using too much energy, this would make an effective method of producing clean energy. However, these ideas would still not work today due to the semiconductors tendency to turn to insulators as temperatures lesser than $100 \mathrm{~K}$ [22]. Our paper has been highly hindered by the lack of information in the field of photovoltaic cells at incredibly low temperature. Continued research in this area is not recommended until these limitations are addressed, which displays hope upon the future of photovoltaic systems.

\section{Conclusion}

Through this paper we have analysed methods of laser cooling, specifically doppler cooling, is unsuitable for improving the efficiency of PV systems it goes beyond the ideal limits of efficiency. Maximum efficiency before PC cells and semiconductors alike exist within the temperature ranges of 100 and $300 \mathrm{~K}$.

Photonic cooling was hypothesised as a possible option to reach higher efficiency conditions if experimental data matched a trend in increasing efficiency as temperatures approach absolute zero, as according to efficiency calculations relevant to Carnot systems. While limited data exists, what we do have shows a decrease or plateau in efficiency before reaching ideal supercool conditions. Photonic supercooling is not a practical solution as they take atoms beyond the temperatures of peak efficiency as atoms in the PV cell lack the energy to be excited to the conduction band.

\section{Acknowledgments}

We would like to acknowledge Jurgen Schulte, Blake Regan, Joshua Prichard and Marin Bell, for the guiding and mentoring they provided. We would also like to acknowledge the students of Energy Science and Technology 2018 who peer reviewed our paper.

\section{References and Notes}

1. Pearce JM. Photovoltaics — a path to sustainable futures. Futures. 2002 Sep 1;34(7):663-74. https://doi.org/10.1016/S0016-3287(02)00008-3

2. Rühle S. Tabulated values of the Shockley-Queisser limit for single junction solar cells. Solar Energy. 2016 Jun 1; 130:139-47. https://doi.org/10.1016/j.solener.2016.02.015

3. Green MA. Third generation photovoltaics: advanced solar energy conversion. Springer Science \& Business Media; 2006 Sep 5.

4. How Does Heat Affect Solar Cell Efficiencies? Available online: https://www.civicsolar.com/support/installer/articles/how-does-heat-affect-solar-panel-efficiencies (accessed on 13th May 2018)

5. Explained: Bandgap. Available online: http://news.mit.edu/2010/explained-bandgap-0723 (accessed on 13th May 2018)

6. Landsberg PT, Markvart T. The Carnot factor in solar-cell theory. Solid-State Electronics. 1998 Apr 6;42(4):657-9. https://doi.org/10.1016/S0038-1101(97)00253-0

7. Sun Fact Sheet. Available online: https://nssdc.gsfc.nasa.gov/planetary/factsheet/sunfact.html (accessed on 13th May 2018) 
8. Sachenko AV, Kryuchenko YV, Kostylyov VP, Bobyl AV, Terukov EI, Abolmasov SN, Abramov AS, Andronikov DA, Shvarts MZ, Sokolovskyi IO, Evstigneev M. Photoconversion in the HIT solar cells: Theory vs experiment. arXiv preprint arXiv:1510.06007. 2015 Oct 20.

9. How do I read specifications of my solar panel https://www.altestore.com/blog/2016/04/how-do-iread-specifications-of-my-solar-panel/ (accessed on 21st May 2018)

10. Markvart T. From steam engine to solar cells: can thermodynamics guide the development of future generations of photovoltaics?. Wiley Interdisciplinary Reviews: Energy and Environment. 2016 Sep 1;5(5):543-69. https://doi.org/10.1002/wene.204

11. Joshi A, Dincer I, Reddy B. Thermodynamic assessment of photovoltaic systems. Solar Energy. 2009;83(8):1139-1149. https://doi.org/10.1016/j.solener.2009.01.011

12. Adams CS, Riis E. Laser cooling and manipulation of neutral particles. The New Optics. 1997:139.

13. Doppler cooling, Wikipedia available online at https://en.wikipedia.org/wiki/Doppler_cooling (accessed 21st May 2018)

14. Boltzmann Constant, Wikipedia, available online: https://en.wikipedia.org/wiki/Boltzmann_constant (accessed 21st May 2018)

15. Nemova G, Kashyap R. Twenty Years of Laser Cooling of Solids. InJournal of Physics: Conference Series 2015 (Vol. 619, No. 1, p. 012037). IOP Publishing.

16. Guenette M. The efficiency of photovoltaic solar cells at low temperatures (Doctoral dissertation, Honours Thesis).

17. Temperature dependence of the energy bandgap http://ecee.colorado.edu/ bart/book/eband5.htm (accessed 21st May 2018)

18. Perl EE, Kuciauskas D, Simon J, Friedman DJ, Steiner MA. Identification of the limiting factors for high-temperature GaAs, GaInP, and $\mathrm{AlGaInP}$ solar cells from device and carrier lifetime analysis. Journal of Applied Physics. 2017 Dec 21;122(23):233102. https://doi.org/10.1063/1.5003631

19. Fesharaki VJ, Dehghani M, Fesharaki JJ, Tavasoli H. The effect of temperature on photovoltaic cell efficiency. InProceedings of the 1stInternational Conference on Emerging Trends in Energy Conservation-ETEC, Tehran, Iran 2011 Nov 20 (pp. 20-21).

20. Laser cooling of semiconductors by annihilating excitations, available at: https://arstechnica.com/science/2013/01/laser-cooling-of-semiconductors-by-annihilating-excitations/ (accessed 21st May 2018)

21. Laser cooling and trapping experiment https://www.colorado.edu/physics/phys3340/phys3340_sp07/CourseInformation/Modern/LaserCooli ng.pdf (accessed 21st May 2018)

22. Kim GH, Shao L, Zhang K, Pipe KP. Engineered doping of organic semiconductors for enhanced thermoelectric efficiency. Nature Materials. 2013 Aug;12(8):719. https://doi.org/10.1038/nmat3635 